\title{
Effects of yoga on performance in a letter-cancellation task under academic examination stress
}

\author{
Dr Bhagaban Bhuyan ${ }^{1}$ Dr Prafulla Kumar Mishra ${ }^{2}$ \\ ${ }^{I}$ Department of Human Consciousness and Yogic Sciences, Utkal University, BBSR, India \\ ${ }^{2}$ Department of Sanskrit, Utkal University, BBSR, India
}

\begin{abstract}
The present study was an attempt to determine whether yoga has an effect on performance in a sixletter cancellation task under examination stress. 122 Class X students between 14 and 17 years of age were randomized into two groups. One group practiced yoga for one hour per day, five days a week, while the other group carried on with their usual routine. Assessments were done before twelve weeks and on the day of examination. Results showed that the total and net scores were significantly higher in both groups. However, the magnitude of change was more in the yoga group than the control group (Total scores $28.93 \%$ versus $7.68 \%$, net scores $30.22 \%$ versus $6.73 \%$ ). There was reduction in scores for wrong cancellation in the yoga group, but not in the control group. The results suggest that yoga improves performance in tasks requiring attention and reduces distraction under academic examination stress.
\end{abstract}

Keywords: attention task; academic examination stress; yoga

\section{Introduction}

Education is one of the most eminent features of the human being resource advancement. One of the major and the natural task for educational professionals, researchers and scientists are to establish a good learning background and to help students get academic success. Examination stress is one of the main obstacles to reach this goal. Examination (exam) is a common short-term stress for students [1, 2]. Seaward, (2002) reported that high level of stress can make more difficult for students to concentrate and grasp information [3]. Many students have the cognitive ability to do well on the exam but may not do so because of high levels of exam stress interfering with their concentration and ability to process information [4]. In addition, students with higher levels of exam stress displayed slower times on attention-measuring tasks than low-anxious students [5].

Many studies have been published analyzing the effect of different aspects of yoga including physical postures (asanas), voluntary regulation of breathing (pranayama), and meditation on attention measuring task. Asanas, pranayama, meditation, cleansing practices (kriyas), devotional sessions and lectures on the theory and philosophy of yoga improve attention span in school children [6]. Sahasi et al. (1989) has demonstrated the effectiveness of yogic techniques in the management of anxiety and reported increased attention and concentration [7]. The studies on different yogic techniques, i.e., deep relaxation technique (DRT) and supine rest (SR) [8], pranayama [9] demonstrated an increase in attention. Two yoga-based relaxation techniques, namely, cyclic meditation (CM) and supine rest (SR) on the six letter cancellation task (SLCT) reported significant increase attention scores in school students [10]. In previous study, SLCT that measured sustained attention in a yoga-based Gurukula education system (GES) compared to a modern education system (MES) found a significant increase in sustained attention scores over the academic year for the GES group $(P<0.05)$, but the increase for the MES group did not reach significance [11]. In another study, Sarang et al. (2007) assessed performance on SLCT in forty male subjects immediately before and after two yoga-based relaxation techniques of CM and SR with equal duration. Both practices significantly improved net scores, total scores $(\mathrm{p}<$ $0.001)$, score for wrong cancellation $(p<0.05)$. CM demonstrated more change $(26 \%)$ than SR $(14 \%)$ in net scores [12]. Meditation showed increases in attention span whereas eyes-closed rest without meditation does not increase attention span [13]. The Self-Regulation Method (SRM) derived from Zen meditation and Autogenic training, which show the state of 'relaxed alertness' also increases attention span [14].

It is known that the yoga techniques bring enhancement in the performance task. So it is a pertinent research question to investigate whether yoga effects on performance in a letter-cancellation task under academic examination stress or not. A cancellation task was chosen as the task is quick measures of attention, concentration, and visuospatial scanning skills [15]. 


\section{Materials And Methods}

\subsection{Subjects}

The study comprises 122 Class X high school students with ages ranging from 14 to 17 years. (Yoga group Mean=15.12year, $\mathrm{SD}=0.62$ and Control group Mean=15.01year, $\mathrm{SD}=0.51$ ). All of them were healthy and without previous exposure to yoga. The participants were excluded from the study if they had a history of neurological or psychiatric disturbances were younger than age 14 or older than 17 years of age, under medication, physical disabilities, had a history of learning disability. The subjects were selected from Ramprasad high school, Chaumukh, and Krushna Chandra High school, Narayanapur, Balasore, Odisha, who satisfied the inclusion and exclusion criteria and randomized into control group (Male-38, Female-28) and yoga group (Male-49, Female-17). After a complete description of the study, the participants had given their written informed consent.

\subsection{Assessments}

The Six Letter Cancellation Task (SLCT) was presented with a worksheet which specified the six target letters to be cancelled and a 'working section' of letters of the alphabet arranged randomly in 22 columns and 14 rows. The participants were asked to cancel as many of the six target letters as possible in the specified time of 1.30 minutes. They were told that there were two possible strategies, i.e., marking all six letters at a time or selecting any one target letter from the six. Participants were asked to choose whichever strategy suited them. They were also told that they could follow a horizontal, vertical, or a random path according to their choice [16]. The assessments were done for both groups, before twelve weeks and on the day of board examination.

Each component of SLCT measures a different measure and function of the brain. The total number of cancellations is a measure of motor skill combined with cognitive function. Net score is a measure of sustained attention which is associated with right frontoparietal cortex [17]. The number of wrong cancellations is a measure of lack of focused attention and mental distraction.

\subsection{Data extraction}

The subjects were seated in a row with enough space between them in a disturbance free room. Proper explanation was given about the tests that were going to be conducted. Pencil and SLTC sheets were provided and instruction was given to all subjects. After clearing their doubts about the tests, the instruction was given to start cancellation. Total time taken for the task was calculated with stopwatch. Time was given 1.30 minutes to do this task. Soon after the test sheets were collected back. Each cancelled letter was scored as ' 1 ' irrespective of whether it was correctly or incorrectly cancelled. The total number of cancellations and wrong cancellations were counted and the net scores were calculated by deducting wrong cancellations from the total cancellations attempted [18]. Scoring was carried out by a person blind to the details of the design of the study.

\subsection{Intervention}

The yoga group followed one hour per day yoga class including yoga postures (asanas), voluntary regulated breathing (pranayama), deep relaxation technique(DRT), meditation (dhyana), and yogic games (a set of games which not only gives stimulation but also relaxation and calms the mind), 5 hours per week, supervised by a trained yoga teacher. While the control group attained normal routine activities along with physical exercises, mathematical puzzles, music, and normal sports.

\subsection{Data analysis}

Statistical analysis was done using SPSS (Version 10.0). The Kolmogorov test of normality showed that the predata were not normally distributed. Hence, nonparametric tests were used for the analysis. Within the groups, the pre-post data were analyzed using the Wilcoxon signed ranks test, while between groups pre-post data were analyzed using the Mann-Whitney U test.

\section{Results}

The Wilcoxon signed ranks test comparing the pre-post values within the groups on the total scores and net scores were significant at $P<0.001$ and the scores for wrong cancellation were significant at $P<0.05$. The MannWhitney $\mathrm{U}$ test used to compare results between the two groups showed a significant difference between the two groups at $P<0.001$. The groups mean values and standard deviations for total scores, net scores and scores for wrong cancellation in the yoga group and the control group are given in Table 1 
TABLE 1

Total scores, net scores and scores for wrong cancellation in six-letter cancellation task before and after yoga group and control group

\begin{tabular}{|c|c|c|c|c|c|c|c|}
\hline \multirow{2}{*}{$\begin{array}{l}\text { Variable and } \\
\text { state }\end{array}$} & \multicolumn{2}{|c|}{ Yoga } & \multirow[b]{2}{*}{$\%$ change } & \multicolumn{2}{|c|}{ Control } & \multirow[b]{2}{*}{$\%$ change } & \multirow{2}{*}{$\begin{array}{l}\begin{array}{l}\text { Between } \\
\text { group } \\
\text { significance }\end{array} \\
\text { On Post scores }\end{array}$} \\
\hline & Mean & S.D. & & Mean & S.D. & & \\
\hline \multicolumn{8}{|l|}{ Total scores } \\
\hline Pre & 33.14 & 8.16 & $28.93 \%$ & 32.8 & 6.92 & $7.68 \%$ & \\
\hline \multicolumn{8}{|l|}{ Net scores } \\
\hline Pre & 32.23 & 7.91 & \multirow{2}{*}{$30.22 \%$} & 32.06 & 6.67 & \multirow{2}{*}{$6.73 \%$} & \multirow{2}{*}{ 0.001@ } \\
\hline Post & $41.97 * *$ & 10.97 & & $34.22 * *$ & 7.46 & & \\
\hline \multicolumn{8}{|c|}{ Scores for wrong cancellation } \\
\hline Pre & 0.92 & 0.73 & $30.43 \%$ & 0.75 & 0.64 & $49.33 \%$ & 0.001@ \\
\hline
\end{tabular}

$* \mathrm{p}<0.05, * * \mathrm{p}<0.001$ Wilcoxon signed ranks test, comparing pre and post values within groups

@ $\mathrm{p}<0.001$ Mann-Whitney U test, comparing between groups

\section{Discussion}

The performance in SLCT was improved in both yoga group and control group. However, the magnitude of change was more in the yoga group than the control group (Total scores $28.93 \%$ versus $7.68 \%$, net scores $30.22 \%$ versus $6.73 \%$ ). There was reduction in the scores for wrong cancellation in the yoga group, but not in the control group. In the present study, the changes in SLCT had a similar trend as in an earlier study, which reported that yoga-based relaxation techniques enhance in the performance task [10].

However, in earlier study there was a $14.76 \%$ in the total score, $14.50 \%$ in the net score improvement. This difference of change could be due to the subjects in the previous study had an average experience only a sevenday yoga training program, while in the present study the subjects had undergone 12 weeks yoga training program. These results revealed that the average duration of the training and practice had an influence on the outcome measures. As in the earlier study, the progressive relaxation technique found modest effects in the first and second weeks before and after the session in the 'Smith Relaxation State Inventory, but major effects were observed in the fourth and fifth weeks of relaxation [19].

Cancellation tasks involve sustained attention, concentration, visual scanning, and activation and inhibition of rapid responses [20]. The findings of the study suggest that the increase attention after yoga in total scores [21], net scores [17] and also reduces distraction [22]. The results also indicated that general participation did not occur being distracted i.e., making errors in the control group. It is known that the yoga techniques bring enhancement in the performance task. In another previous study demonstrated that yoga can lead to additional improvement in cognitive functions, such as, remote memory, mental balance, attention and concentration, delayed and immediate recall, verbal retention and recognition tests in climacteric syndrome [23].

Earlier study has shown anxiety affects performance on task requiring attention [24]. Yoga practices have been found to rectify the under activity of the parasympathetic nervous system (PNS) and gamma-amino butyric acid (GABA) systems in part through stimulation of the vagus nerves, the main peripheral pathway of the PNS [25] and yoga-based guided relaxation and meditation has also been reported to reduce sympathetic activity, balance neuro-endocrine axis and decrease anxiety and stress levels [26, 27], could have facilitated increase attention task performance.

\section{Conclusion}

It may be concluded from the findings of the study that yoga improves performance on task requiring attention and reduces distraction under academic exam stress.

The students were assessed twice with an interval of 12 weeks. Limitation of the study design was short duration and no follow-up. Another limitation of the study was the student's anxiety and stress levels were not measured. Further studies could be designed to assess this.

Yoga is ancient Indian science which is an inexpensive and simple method to help mitigate the deleterious effect stress, anxiety and enhance the performance on tasks requiring attention. Therefore, it is suggested that yoga module should become a regular feature of the school curriculum. 


\section{References}

[1] K. Vedhara, J. Hyde, I.D. Gilchrist, M. Tytherleigh, and S. Plummer, Acute stress, memory, attention and cortisol, Psychoneuroendocrinology, 25, 2000, 535-549.

[2] M.S. Chapell, Z.B. Blanding, and M. E. Silverstein, Test- anxiety and academic achievement in undergraduate and graduate students, Journal of Education Psychology, 97(2), 2005, 268-278.

[3] B. L. Seaward, Managing stress: Principles and strategies for health and well-being. (Boston, MA: Jones and Bartlett Publishers, 2002).

[4] M. Zeidner, Does test anxiety bias scholastic aptitude test performance by gender and socio-cultural group? Journal of Personality Assessment, 55, 1990, 145-60.

[5] S. Lindsay, The effect of test anxiety on attention and memory skills in undergraduate students. Chrestomathy: Annual Review of Research at the College of Charleston, USA, 1, 2002, 263-273.

[6] S. Telles, B. Hanumanthaiah, R. Nagarathna, and H.R. Nagendra, Improvement in static motor performance following yogic training of school children, Percept Motor Skills, 76, 1993, 1264-6.

[7] G. Sahasi, D. Mohan, and C. Kacker, Effectiveness of yogic techniques in the management of anxiety, Journal of Personality and Clinical Studies, 1, 1989, 51-5.

[8] S.S. Khemka , N.H. Rao, and R. Nagarathna, Immediate effects of two relaxation techniques on healthy volunteers, Indian $J$ Physiol Pharmacol, 53(1), 2009,67-72.

[9] S. Telles, P. Raghuraj, S. Maharana, and H.R. Nagendra, Immediate effect of three breathing techniques on performance in a letter cancellation task. Psychological reports, 104, 2007, 1289-1296.

[10] P. Balaram, and H. R. Nagendra, Immediate effect of two yoga-based relaxation techniques on attention in children, Int $J$ Yoga, 3(2), 2010, 67-69.

[11] R. Rangan, H.R. Nagendra, and Ramachandra Bhatt, Effect of yogic education system and modern education system on sustained attention, Int $J$ Yoga, 2(1), 2009, 35-38.

[12] P.S. Sarang, and S. Telles, Immediate effect of two yoga-based relaxation techniques on performance in a letter cancellation task, Perceptual and Motor Skills, 105, 2007, 379-385.

[13] J. P. Banquet, J.C. Bourzeix, and N. Lesevre, Evoked potentials and vigilance states induced during the course of choice reaction time tests, Rev Electroencephalogr Neurophysiol Clin, 9(3), 1979, 221-7.

[14] A. Ikemi, S. Tomita, M. Kuroda, Y. Hayashida, and Y. Ikemi, Self-regulation method: Psychological, physiological and clinical considerations: An overview, Psychother Psychosom, 46(4), 1986, 184-95.

[15] B. Uttl, and C. Pilkenton-Taylor, Letter cancellation performance across the adult life span, Clinical Neuropsychology, 15, 2001, 521-530.

[16] M. V. Natu, and A. K. Agarawal, Testing of stimulation effects of coffe on the psychomotor performance: an exercise in clinical pharmacology, Indian Journal of Pharmacology, 29, 1997, 11-14.

[17] I. Rueckert, and J. Graffman, Sustained attention deficits space in patients with right frontal lesions. Neuro Psychologia, 36, 1996, 953-63.

[18] A.K. Agarwal, R. Kalra, M.V. Natu, A.P. Dadich, and R.S. Deswal, Psychomotor performance of psychiatric inpatients under therapy: Assessment by paper and pencil tests, Hum Psychopharmacol, 17(2), 2002, 91-3.

[19] M. Matsumoto, and J. C.Smith, Progressive muscle relaxation, breathing exercise, and ABC relaxation theory, J Clin Psycol, 57 , $2001,1551-7$.

[20] M. Lezak, D. B.Howieson, and D. W. Loring, Neuropsychological assessment, (New York: Oxford University Press, 2004).

[21] J. M. Fuster, The prefrontal cortex anatomy, physiology and neurophysiology of the frontal lobe. (2nd Ed. New York: Raven Press, 1989).

[22] L.S. Rao, D.K. Subbakrishna, and K. Gopukumar, NIMHANS Neuro-psychology battery-2004 manual, (The national institute of mental health and neurosciences, Bangalore, India, 2004).

[23] R. Chattha, R.Nagarathna, V.Padmalatha, and H.R. Nagendra, Effect of yoga on cognitive functions in climacteric syndrome: a randomized control study, BJOG, 115(8), 2008, 991-1000.

[24] E. Fox, Attentional bias in anxiety: selective or not? Behavioral Research and Therapy, 31(5), 1993, 487-93.

[25] C.C. Streeter, P.L. Gerbarg, R.B. Saper, D.A. Ciraulo, and R. P. Brown, Effects of yoga on the autonomic nervous system, gam ma-aminobutyric-acid, and allostasis in epilepsy, depression, and post-traumatic stress disorder, Med Hypothesis, 78(5), 201 2, 571-579.

[26] R. P. Vempati, and S. Telles S, Yoga-based guided relaxation reduces sympathetic activity judged from base line levels, Psychol Rep, 90, 2002, 487-494.

[27] S. H. Lee, S. C. Ahn, Y. J. Lee, T. K. Choi, K. H. Yook, and S. Y. Shu, Effectiveness of a meditation-based stress management program as an adjunct to pharmacotherapy in patients with anxiety disorder, J Psychosom Res, 62, 2007, 189-195 